\title{
Desvenlafaxine Versus Placebo in a Fluoxetine-Referenced Study of Children and Adolescents with Major Depressive Disorder
}

\author{
Karen L. Weihs, MD, William Murphy, $\mathrm{MD}^{2}$, Richat Abbas, $\mathrm{PhD}^{3}$, Deborah Chiles, BA, RN, \\ Richard D. England, MD, PhD, Sara Ramaker, BA, and Dalia B. Wajsbrot, $\mathrm{MSc}^{5}$
}

\begin{abstract}
Objectives: To evaluate the short-term efficacy and safety of desvenlafaxine $(25-50 \mathrm{mg} / \mathrm{d})$ compared with placebo in children and adolescents with major depressive disorder (MDD).

Methods: Outpatient children (7-11 years) and adolescents (12-17 years) who met DSM-IV-TR criteria for MDD and had screening and baseline Children's Depression Rating Scale-Revised (CDRS-R) total scores $>40$ were randomly assigned to 8 -week treatment with placebo, desvenlafaxine $(25,35$, or $50 \mathrm{mg} / \mathrm{d}$ based on baseline weight), or fluoxetine $(20 \mathrm{mg} / \mathrm{d})$. The primary efficacy endpoint was change from baseline in CDRS-R total score at week 8 , analyzed using a mixed-effects model for repeated measures. Secondary efficacy endpoints included week 8 Clinical Global Impressions-Severity, Clinical Global Impressions-Improvement (CGI-I), and response (CGI-I $\leq 2)$. Safety assessments included adverse events, physical and vital sign measurements, laboratory evaluations, electrocardiogram, and the Columbia-Suicide Severity Rating Scale.

Results: The safety population included 339 patients (children, $n=130$; adolescents, $n=209$ ). The primary endpoint, change from baseline in CDRS-R total score at week 8 , did not statistically separate from placebo, for either desvenlafaxine (adjusted mean [standard error] change, -22.6 [1.17]) or fluoxetine (-24.8 [1.17]; placebo, -23.1 [1.18]). Week 8 CGI-I response rates were significantly greater for fluoxetine $(78.2 \% ; p=0.017)$ than for placebo $(62.6 \%)$; desvenlafaxine $(68.7 \%)$ did not differ from placebo. Other secondary outcomes were consistent with those obtained with CDRS-R. Rates of treatment-emergent adverse events were comparable among treatment groups (desvenlafaxine, 60.0\%; placebo, 70.5\%; and fluoxetine, 64.3\%). Conclusion: Desvenlafaxine did not demonstrate efficacy for treating MDD in children and adolescents in this trial. Because neither desvenlafaxine nor the reference medication, fluoxetine, demonstrated a statistically significant difference from placebo on the primary endpoint, this was considered a failed trial and no efficacy conclusions can be drawn. Desvenlafaxine $25-50 \mathrm{mg} / \mathrm{d}$ was generally safe and well tolerated in children and adolescents in this study.
\end{abstract}

Keywords: desvenlafaxine, major depressive disorder, treatment efficacy, clinical trial, children, adolescents

\section{Introduction}

$\mathbf{P}$ REVALENCE RATES FOR major depressive disorder (MDD) have been estimated at up to $2.5 \%$ in children and $8.3 \%$ in adolescents (Birmaher et al. 1996). Untreated MDD in children and adolescents can affect family interactions, social development, and social functioning, as well as school performance (Birmaher et al. 1996, 2007; Siu 2016). For these reasons, screening for MDD in primary care settings is recommended for all pediatric patients aged $12-18$ years
(Siu 2016). The standard of care for treatment of moderate-to-severe depression in adolescents and children is psychological intervention, an antidepressant medication, or both (Birmaher et al. 2007; Cheung et al. 2007).

Several antidepressant medications have been assessed for efficacy for treating MDD in pediatric populations, including selective serotonin reuptake inhibitors (SSRIs) and serotonin-norepinephrine reuptake inhibitors (SNRIs) (Emslie et al. 1997, 2002, 2007, 2009, 2014; Wagner et al. 2003, 2004; Atkinson et al. 2014). Fluoxetine

\footnotetext{
${ }^{1}$ Department of Psychiatry, University of Arizona, Tucson, Arizona.

${ }^{2}$ Psychiatric Associates, Overland Park, Kansas.

${ }^{3}$ Pfizer Inc, Collegeville, Pennsylvania.

${ }^{4}$ Pfizer Inc, Groton, Connecticut.

${ }^{5}$ Pfizer Inc, New York, New York.

Funding: This study was sponsored by Pfizer Inc.
}

(c) Karen L. Weihs et al. 2017; Published by Mary Ann Liebert, Inc. This article is available under the Creative Commons License CC-BY-NC (http:// creativecommons.org/licenses/by-nc/4.0). This license permits non-commercial use, distribution and reproduction in any medium, provided the original work is properly cited. Permission only needs to be obtained for commercial use and can be done via RightsLink. 
demonstrated statistical superiority over placebo in studies that enrolled children and adolescents (Emslie et al. 1997, 2002) and is approved for the treatment of pediatric MDD in many countries, including the United States (Prozac [package insert] 2014). Escitalopram, which had significant efficacy compared with placebo in patients aged 12-17 years (Emslie et al. 2009), is approved for the treatment of MDD in adolescents (Lexapro [package insert] 2014). However, for patients who do not tolerate or respond fully to those medications, additional antidepressant therapy options are needed.

The SNRI desvenlafaxine (administered as desvenlafaxine succinate) has demonstrated safety, tolerability, and efficacy in randomized, double-blind, placebo-controlled studies in adult patients with MDD (DeMartinis et al. 2007; Septien-Velez et al. 2007; Boyer et al. 2008; Liebowitz et al. 2008; Tourian et al. 2009; Dunlop et al. 2011; Clayton et al. 2013, 2015; Iwata et al. 2013) and is approved for the treatment of adults with MDD, with a recommended therapeutic dose of $50 \mathrm{mg} / \mathrm{d}$ (Pristiq [package insert] 2016). No significant efficacy compared with placebo has been observed for desvenlafaxine doses below $50 \mathrm{mg} / \mathrm{d}$ (Iwata et al. 2013; Liebowitz et al. 2013). The safety and tolerability of desvenlafaxine was examined in pediatric patients ages 7-17 with MDD in an 8-week, open-label, fixed-dose phase IIa study of doses ranging from 10 to $200 \mathrm{mg} / \mathrm{d}$ and a 6-month extension study, in which desvenlafaxine treatment appeared to be generally safe and well tolerated (Findling et al. 2014). Neither study, however, included a placebo arm. To effectively examine the efficacy and safety of desvenlafaxine for the treatment of pediatric patients with MDD, the sponsor (Pfizer Inc) planned four phase III studies, including two shortterm and two 6-month extension studies. The pharmacokinetic profile of desvenlafaxine over a 10- to 200-mg dose range, determined based on samples collected during the phase IIa study (Findling et al. 2016), informed the dosing design of the phase III program. This study (NCT01372150) is the first of two similarly designed, short-term, double-blind, placebo-controlled studies of desvenlafaxine for the treatment of MDD in children and adolescents. Results from the second short-term study and extension studies will be reported separately.

The objectives of this short-term, placebo-controlled, and fluoxetine-referenced study were to evaluate the efficacy, safety, and tolerability of desvenlafaxine in the treatment of children and adolescents with MDD.

\section{Methods}

Patients were randomized at 37 sites; 35 sites were in the United States (1-31 patients per site; median, 7) and 2 in Mexico (13 and 17 patients). Study sites included hospitals and academic institutions (5), clinical/private practices (3), and clinical trial research centers (29). Hospitals and academic institutions enrolled 41/340 patients (12\%). Principal investigators at each site were child and adolescent psychiatrists (20) or general psychiatrists (17), who were board eligible or board certified (or completion of an equivalent training program for Mexican investigators), with experience in the diagnosis and treatment of pediatric depression and in conducting industrysponsored studies. The study was conducted between November 2011 and March 2015 and in accordance with the International Council for Harmonisation Guideline for Good Clinical Practice (International Council for Harmonisation 1998) and the ethical principles that have their origin in the Declaration of Helsinki. The study protocol received institutional review board or independent ethics committee approval. Written informed consent and assent were obtained from legal guardians and participants before any study procedures were performed. Periodic safety review was conducted by an independent Data Monitoring Committee.

\section{Study design}

This was a phase III, multicenter, randomized, double-blind, placebo-controlled, fluoxetine-referenced, 8-week, parallel group study. Patients who completed this acute-phase study were eligible to participate in a 6-month, open-label extension study of desvenlafaxine. Patients were randomly assigned (1:1:1) to placebo, desvenlafaxine, or fluoxetine, stratified by age group (child or adolescent) and country. Eight-week double-blind treatment was followed by a 1-week double-blind transition phase for patients who entered the extension study or a 1-week double-blind taper phase for those who did not.

The selection of doses was based on two factors: first, the highest dose used in the study was $50 \mathrm{mg} / \mathrm{d}$ because no efficacy benefit has been demonstrated at doses higher than $50 \mathrm{mg} / \mathrm{d}$ in adults and tolerability decreases at doses higher than $50 \mathrm{mg} / \mathrm{d}$ (Thase et al. 2009). The recommended desvenlafaxine dose is $50 \mathrm{mg} / \mathrm{d}$ in adults with MDD (Pristiq [package insert] 2016). In studies of adults with MDD, no dose response for efficacy was observed for desvenlafaxine doses of 50 to $400 \mathrm{mg} / \mathrm{d}$; however, a dose response for safety was demonstrated (Clayton et al. 2009; Thase et al. 2009). The $50 \mathrm{mg} / \mathrm{d}$ dose was generally safe and well tolerated in a phase IIa study in children and adolescents with MDD (Findling et al. 2016). Second, pharmacokinetic data from the phase IIa study demonstrated that body weight predicts desvenlafaxine exposure in a pediatric MDD population (Findling et al. 2016). Desvenlafaxine dose was therefore assigned based on the patient's body weight at the baseline (day 1) visit, with $50 \mathrm{mg} / \mathrm{d}$ as the highest dose, as follows: 20 to $<35 \mathrm{~kg}$ : $25 \mathrm{mg} / \mathrm{d} ; 35$ to $<70 \mathrm{~kg}$ : $35 \mathrm{mg} / \mathrm{d}$; and $\geq 70 \mathrm{~kg}$ : $50 \mathrm{mg} / \mathrm{d}$. Titration, taper, and transition dosing is shown in Supplementary Table S1 (Supplementary Data are available online at www.liebertpub.com/cap).

\section{Study patients}

Study participants were male and female outpatients, aged 7 to $<18$ years who weighed at least $20 \mathrm{~kg}$ at the screening and baseline visits. All enrolled patients met Diagnostic and Statistical Manual of Mental Disorders, 4th edition (Text Revision) (DSM-IV-TR) (American Psychiatric Association 2000) criteria for MDD as the primary diagnosis, had depressive symptoms of at least moderate severity for at least 30 days, and would not require concomitant psychotherapy. The MDD diagnosis was confirmed by a psychiatrist at the study site and supported by the KIDDIE Schedule for Affective Disorders and Schizophrenia-Present and Lifetime Version (K-SADS-PL). Enrolled patients had a Children's Depression Rating Scale-Revised (CDRS-R) total score $>40$ and Clinical Global Impressions Scale-Severity (CGI-S) (Guy 1976) score of 4 or greater at screening and baseline. Patients and their parents/guardians provided informed consent and assent.

Patients were excluded from the study if they: had a history or presence of MDD with psychotic features or any psychotic disorder, bipolar disorder (or first-degree relative with bipolar disorder) or manic episodes or comorbid primary psychiatric condition other than MDD, or a history of or current significant risk of suicide, or first-degree relative who had committed suicide. Detailed exclusions and prohibited treatments are listed in Supplementary Data S1.

\section{Assessments}

Efficacy. Efficacy assessments were administered at weeks $1,2,3,4,6,8$, and/or at early termination in the double-blind phase. 
A week 9 assessment was administered after taper or after transition as the baseline assessment for the extension study for those who were continuing. The primary efficacy outcome was change from baseline in the CDRS-R (Poznanski et al. 1979) total score at week 8. The protocol-defined key secondary efficacy outcome was change from baseline in CGI-S score; other secondary efficacy outcomes were change from baseline in Clinical Global Impressions Scale-Improvement (CGI-I) (Guy 1976) score and CGI-I response (CGI-I score $\leq 2$ ) at each visit. Site personnel administering the K-SADS-PL, CDRS-R, CGI-S, and CGI-I were qualified (with a minimum of 2 years' clinical experience with pediatric MDD), trained by the study sponsor, and approved as evaluators before conducting the assessments. Those administering the K-SADS-PL and CGI were further required to be either a doctoral level psychologist or a psychiatrist and have at least 2 years' experience using the scale. Raters for the CDRS-R were required to be either a doctoral or nondoctoral (e.g., MA, MS, MSW, RN, BS, BA) level clinician or a psychiatrist, to have at least 2 years' experience using the scale, and to be certified by the sponsor. Certification was a two-step process: raters had to (1) meet predefined interrater reliability criteria against the gold standard scores using videotaped assessments and (2) complete a one-on-one training on CDRS-R interview technique (applied training) through the rater training vendor, achieving acceptable technique and reliability in accordance with prespecified criteria using the Rater Applied Performance Scale (Kobak et al. 2005). The protocol recommended that, whenever possible, the same rater administered a given scale for the patient at each assessment.

Safety. Adverse event (AE; MedDRA v17.1) assessments, vital sign measurements, and Columbia-Suicide Severity Rating Scale (C-SSRS) (Posner et al. 2011) assessments were performed at each study visit. A physical examination with Tanner assessment and laboratory evaluations were performed at screening and week 8 , with liver function tests and serum lipids also evaluated at week 4; ECG was performed at screening, baseline, and week 8 . Individuals completing the C-SSRS and Tanner assessments were qualified, trained by the study sponsor, and approved as evaluators before conducting those assessments. The protocol recommended that, whenever possible, the same rater administer the Tanner for the patient at each assessment.

Serious AEs were defined as any untoward medical occurrence at any dose of study medication that resulted in death or events that posed immediate risk of death, required inpatient hospitalization or prolongation of hospitalization, resulted in persistent or significant disability/incapacity, or resulted in congenital anomaly/birth defect. Important medical events were also reported as serious AEs when it was determined that they may have jeopardized the patient or required intervention to prevent one of the other serious $\mathrm{AE}$ outcomes. Potentially clinically important (PCI) findings were identified based on changes in laboratory findings, vital signs, and ECG results defined according to criteria prespecified by the sponsor. Clinically important results were then identified by the medical monitor based on a review of patient data, relevant clinical information pertaining to a patient in case report forms, and clinical judgment.

Pharmacokinetics. Pharmacokinetic samples were collected at weeks 4 and 8 to support an integrated population pharmacokinetic analysis and an exploratory concentration-effect analysis. The results of these analyses will be reported and published separately.

\section{Statistical analysis}

Sample size determination. The sample size estimate was performed for the change from baseline in CDRS-R total score at week 8. A sample of 111 patients per group was considered sufficient to demonstrate a 5-point difference in the primary endpoint between the desvenlafaxine and placebo groups at a significance level of $5 \%$ and a power of $85 \%$, assuming a pooled standard deviation (SD) of 12 , and that no more than $5 \%$ of randomized subjects would fail to qualify for the primary analysis $(<1 \%$ in the current study). Results of a planned interim analysis (Supplementary Data S2) indicated that no sample size increase was required.

Efficacy. Efficacy analyses were based on the intent-to-treat (ITT) population: all patients who were randomly assigned to treatment received at least one dose of study medication, and had a baseline and at least one postbaseline primary efficacy assessment. The analysis of the change from baseline in CDRS-R total score was performed using a mixed-effects model for repeated measures with terms for treatment, week, interaction of treatment and week, age group, country, gender, and baseline CDRS-R total score. Statistical superiority of desvenlafaxine over placebo was to be declared based on a $p$-value $<0.05$. Desvenlafaxine and fluoxetine were separately compared with placebo. Fluoxetine was included as an active reference to provide assay sensitivity only and was not compared directly with desvenlafaxine. Sensitivity analyses are described in Supplementary Data S2.

The CGI-S score was analyzed using the same approach as the CDRS-R total score. The categorical CGI-I score was analyzed at each visit using the Cochran-Mantel-Haenszel (CMH) row-meanscore-difference test using ridit scores and controlling for age/ country stratum. CGI-I response rates were analyzed at each visit using a logistic regression model with terms for treatment, age group, and country.

Safety. Safety summaries were presented for the safety population, defined as all patients who were randomly assigned to treatment and received at least one dose of study medication. The incidence rates for prespecified AEs of clinical importance were compared between treatment groups using risk difference versus placebo and associated $p$-values without multiplicity adjustment. Confidence intervals for the risk difference were calculated using the method by Chan and Zhang (1999).

\section{Results}

\section{Study population}

A total of 340 patients were randomly assigned to treatment; 339 patients (children, $n=130$; adolescents, $n=209$ ) were included in the safety population (desvenlafaxine, $n=115$; fluoxetine, $n=112$; placebo, $n=112$ ). The ITT population included 130 children and 207 adolescents. A total of $42(12 \%)$ patients discontinued early (desvenlafaxine, 16 [14\%]; fluoxetine, 13 [12\%]; and placebo, 13 [12\%]). Lost to follow-up was the most common reason for discontinuation from the desvenlafaxine and placebo groups; in the fluoxetine group, the most common reason for discontinuation was "no longer willing to participate" (Fig. 1).

Demographic and baseline characteristics were comparable among treatment groups for both children and adolescents (Table 1). Mean (SD) CDRS-R Total Score at baseline was 56.5 (8.9), mean (SD) CGI-S score was 4.5 (0.6), and the duration of the most recent depressive episode ranged from 1 to 96 months (median, 7; 


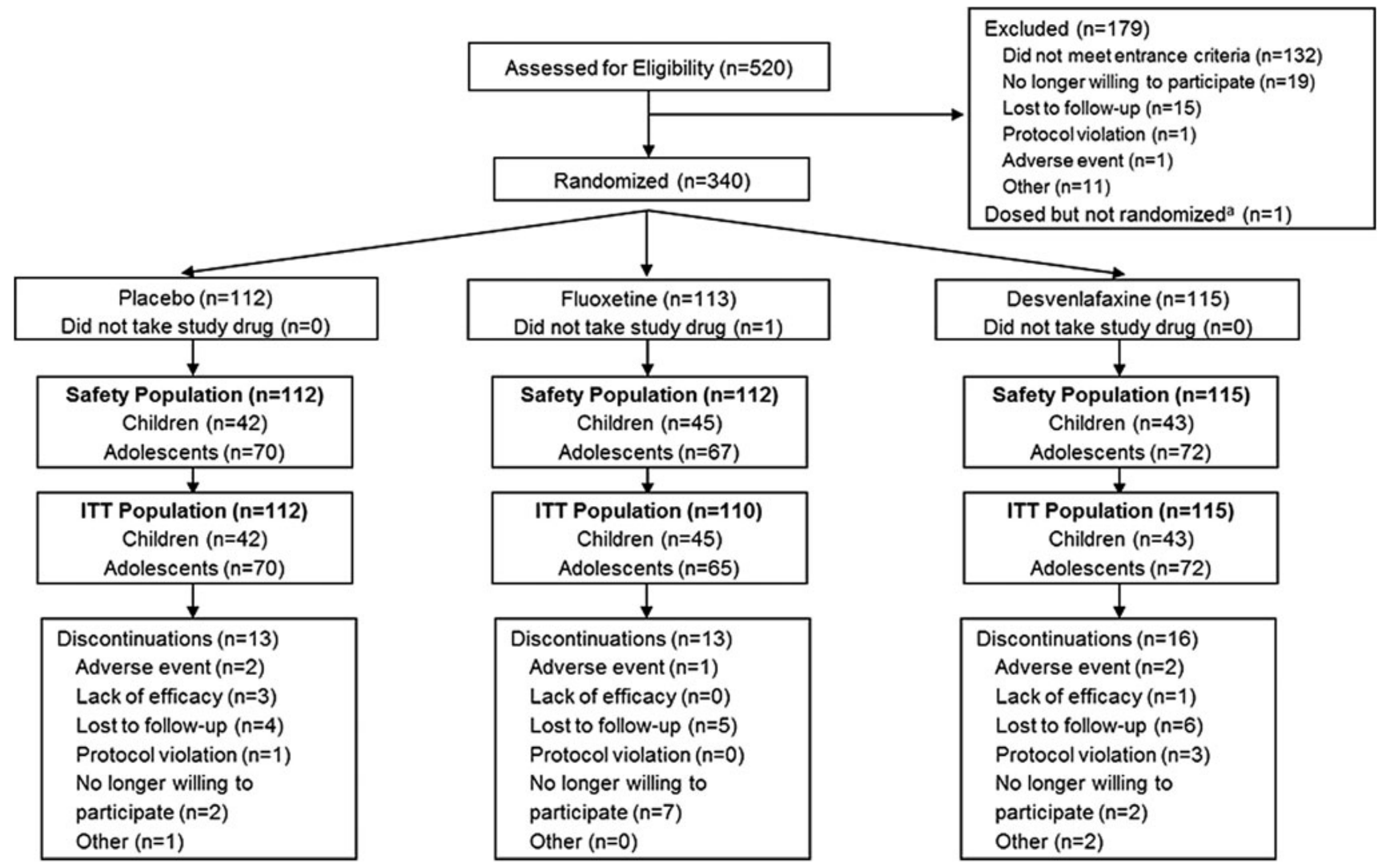

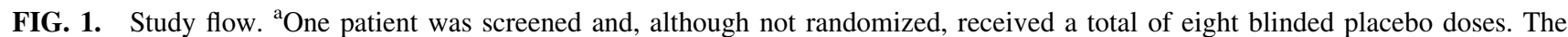
patient was discontinued after the week 1 visit once the error was identified and listed as a protocol violation.

Table 1. Demographic and Baseline Characteristics, Safety Population

\begin{tabular}{|c|c|c|c|c|c|c|c|c|}
\hline & \multicolumn{4}{|c|}{ Children } & \multicolumn{4}{|c|}{ Adolescents } \\
\hline & $\begin{array}{l}\text { Placebo } \\
(\mathrm{n}=42)\end{array}$ & $\begin{array}{l}\text { Fluoxetine } \\
(\mathrm{n}=45)\end{array}$ & $\begin{array}{l}\text { Desvenlafaxine } \\
\quad(\mathrm{n}=43)\end{array}$ & $\begin{array}{c}\text { Total } \\
(\mathrm{n}=130)\end{array}$ & $\begin{array}{l}\text { Placebo } \\
(\mathrm{n}=70)\end{array}$ & $\begin{array}{l}\text { Fluoxetine } \\
(\mathrm{n}=67)\end{array}$ & $\begin{array}{l}\text { Desvenlafaxine } \\
\quad(\mathrm{n}=72)\end{array}$ & $\begin{array}{c}\text { Total } \\
(\mathrm{n}=209)\end{array}$ \\
\hline $\begin{array}{l}\text { Age, mean } \\
\text { (SD), years }\end{array}$ & $9.4(1.3)$ & $9.6(1.3)$ & $9.3(1.4)$ & $9.4(1.3)$ & $14.6(1.5)$ & $14.7(1.6)$ & $15.0(1.5)$ & $14.8(1.5)$ \\
\hline \multicolumn{9}{|l|}{ Sex, $n(\%)$} \\
\hline Female & $23(55)$ & $14(31)$ & $20(47)$ & $57(44)$ & $41(59)$ & $43(64)$ & $43(60)$ & $127(61)$ \\
\hline Male & $19(45)$ & $31(69)$ & $23(53)$ & $73(56)$ & $29(41)$ & $24(36)$ & $29(40)$ & $82(39)$ \\
\hline \multicolumn{9}{|l|}{ Race, $n(\%)$} \\
\hline Asian & $1(2)$ & 0 & $1(2)$ & $2(2)$ & 0 & $2(3)$ & 0 & $2(1)$ \\
\hline Black & $15(36)$ & $15(33)$ & $14(33)$ & $44(34)$ & $10(14)$ & $19(28)$ & $18(25)$ & $47(22)$ \\
\hline White & $25(60)$ & $26(58)$ & $23(53)$ & $74(57)$ & $56(80)$ & $41(61)$ & $49(68)$ & $146(70)$ \\
\hline Other & $1(2)$ & $4(9)$ & $5(12)$ & $10(8)$ & $4(6)$ & $5(7)$ & $5(7)$ & $14(7)$ \\
\hline $\begin{array}{l}\text { Height, mean } \\
\text { (SD), cm }\end{array}$ & $140.5(10.6)$ & $141.3(11.2)$ & $144.3(11.5)$ & $142.0(11.1)$ & $164.5(9.8)$ & $165.8(8.4)$ & $167.3(9.1)$ & $165.9(9.2)$ \\
\hline $\begin{array}{l}\text { Weight, mean } \\
\text { (SD), kg }\end{array}$ & $39.4(14.0)$ & $44.0(18.9)$ & $46.0(15.2)$ & $43.2(16.3)$ & $68.0(19.1)$ & $73.3(21.8)$ & $71.7(19.0)$ & $71.0(20.0)$ \\
\hline $\begin{array}{l}\text { BMI, mean } \\
\quad(\mathrm{SD}), \mathrm{kg} / \mathrm{m}^{2}\end{array}$ & $19.5(4.7)$ & $21.3(6.4)$ & $21.6(5.0)$ & $20.8(5.5)$ & $25.0(6.0)$ & $26.5(6.9)$ & $25.5(5.8)$ & $25.6(6.3)$ \\
\hline $\begin{array}{l}\text { Duration of most } \\
\text { recent episode, } \\
\text { median (range), } \\
\text { months }\end{array}$ & $11(1-57)$ & $6(1-42)$ & $8(1-71)$ & $7(1-71)$ & $8(1-69)$ & $7(1-96)$ & $7(1-61)$ & $7(1-96)$ \\
\hline $\begin{array}{l}\text { CDRS-R total } \\
\text { score, } \\
\text { mean }(\mathrm{SD})\end{array}$ & $57.0(8.6)$ & $55.0(8.7)$ & $56.4(10.9)$ & $56.1(9.4)$ & $57.1(9.1)$ & $57.0(8.1)$ & $56.3(8.8)$ & $56.8(8.7)$ \\
\hline
\end{tabular}

BMI, body mass index; CDRS-R, Children's Depression Rating Scale-Revised; SD, standard deviation. 
mean [SD], 13.4 [15.1]). Overall, 30.4\% (103) of patients in the safety population had a prior or active psychiatric condition other than MDD in their medical history (desvenlafaxine, 31.3\%; fluoxetine, $25.9 \%$; and placebo, $33.9 \%$ ). The most common psychiatric conditions (reported by $\geq 2 \%$ of patients) included attentiondeficit/hyperactivity disorder (desvenlafaxine, $12.2 \%$; fluoxetine, $13.4 \%$; and placebo, 5.4\%), nonsuicidal self-injurious behavior (desvenlafaxine, $7.0 \%$; fluoxetine, $8.0 \%$; and placebo, $12.5 \%$ ), insomnia (desvenlafaxine, $7.0 \%$; fluoxetine, $6.3 \%$; and placebo, $8.0 \%$ ), generalized anxiety disorder (desvenlafaxine, $0.9 \%$; fluoxetine, $6.3 \%$; and placebo, 0), and oppositional defiant disorder (desvenlafaxine, $3.5 \%$; fluoxetine, $3.6 \%$; and placebo, 0 ).

\section{Efficacy}

Statistical separation from placebo was not observed on the primary efficacy endpoint for either desvenlafaxine or for the fluoxetine reference group. Adjusted mean (standard error) change from baseline in CDRS-R total score at week 8 was -22.6 (1.17) and -23.1 (1.18) for the desvenlafaxine and placebo groups, respectively, and for fluoxetine was -24.8 (1.17) (Fig. 2). The change from baseline in CDRS-R over the 8-week treatment phase was similar for the three arms. Point differences were statistically significant between desvenlafaxine and placebo at week 2 and between fluoxetine and placebo at weeks 1 and 2; no significant differences were observed at any other time points. Results were similar for the child and adolescent groups in an exploratory analysis of change from baseline in CDRS-R total score (Supplementary Fig. S1).

At week 8, the CGI-I response rate for fluoxetine (78.2\%) was significantly greater compared with placebo $(62.6 \% ; p=0.017)$. Week 8 CGI-I response rate for desvenlafaxine (68.7\%) was also greater than placebo, although this comparison did not reach statistical significance $(p=0.343)$. Results for CGI-S and CGI-I scores were otherwise consistent with those for CDRS-R total score, with no statistically significant difference from placebo for either desvenlafaxine or fluoxetine at week 8 (Table 2).

\section{Safety}

Adverse events. A total of 220/339 (64.9\%) patients experienced AEs during the on-therapy period (desvenlafaxine, 69/115
[60.0\%]; fluoxetine, 72/112 [64.3\%]; and placebo, 79/112 [70.5\%]). Most AEs were mild or moderate in severity. AEs considered by the investigator to be related to study medication were reported by $31.9 \%$ of patients (desvenlafaxine, $28.7 \%$; fluoxetine, $32.1 \%$; and placebo, $34.8 \%$ ). Severe AEs (those that interfered significantly with patients' usual function) considered by the investigator to be unrelated to study medication were reported by $3.5 \%$ of patients (desvenlafaxine, 1.7\%; fluoxetine, 5.4\%; and placebo, 3.6\%). In three patients, severe AEs were considered related to study medication: One desvenlafaxine-treated patient reported severe pruritus and severe macular rash and discontinued treatment. One placebotreated patient reported severe agitation and severe anger, and a second reported severe insomnia; neither was discontinued due to those AEs. Five adolescents ( 0 children) discontinued due to AEs, two each from the desvenlafaxine (disinhibition; macular rash and pruritus) and placebo (vomiting; headache) groups and one from the fluoxetine group (suicidal ideation).

Treatment-emergent adverse events. Treatment-emergent adverse events (TEAEs) reported by more than $10 \%$ of patients in any group were headache, upper abdominal pain, and nausea. Table 3 shows the most frequent ( $\geq 5 \%$ in any group) TEAEs by age group, and overall. There were no statistically significant differences between desvenlafaxine or fluoxetine and placebo in the incidence of any prespecified TEAEs of clinical interest for desvenlafaxine (tier-1 TEAEs) (Supplementary Table S2).

Deaths and serious AEs. There were no deaths during the study. Five randomized patients experienced serious AEs: two assigned to fluoxetine (suicidal ideation [acute suicidality; discontinued] and suicide attempt [occurred poststudy], one patient each), three assigned to desvenlafaxine (suicidal ideation [discontinued due to lack of confidence in caregiver reliability to report patient status], disinhibition [discontinued], and postpartum hemorrhage with anemia and endometritis [occurred poststudy], one patient each), and no placebo-treated patients. The desvenlafaxinetreated patient with postpartum hemorrhage had been withdrawn from the study following a positive pregnancy test at the final treatment phase visit and delivered a healthy full-term infant.

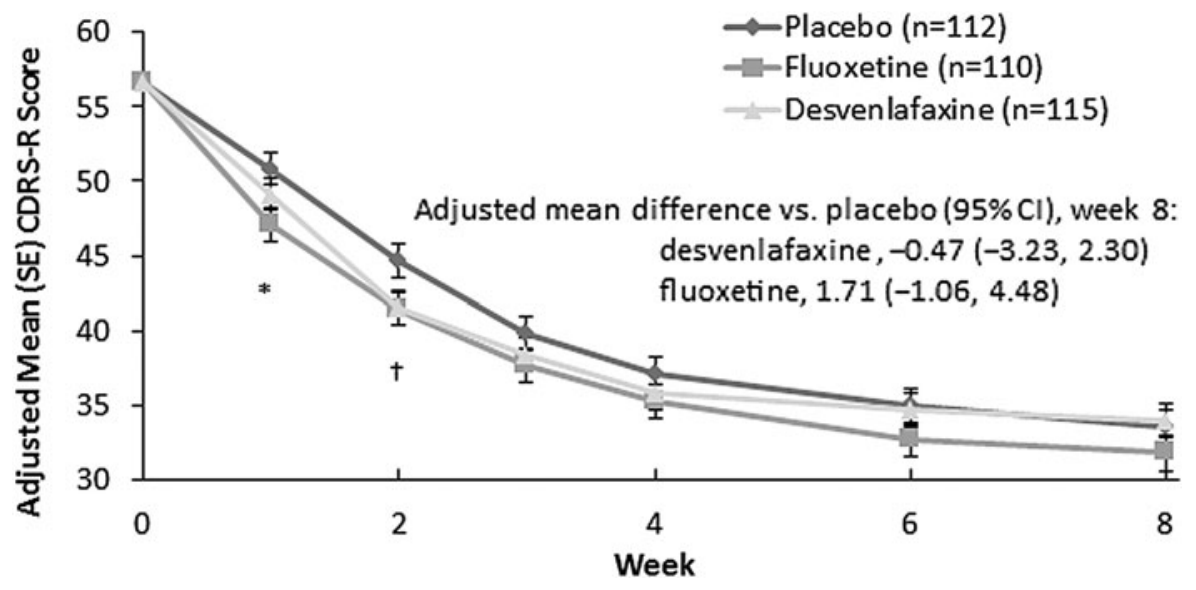

FIG. 2. Adjusted mean (SE) change from baseline in CDRS-R total score in children and adolescents; MMRM analysis, ITT population. ${ }^{*} p<0.01$, fluoxetine versus placebo. ${ }^{\dagger} p<0.05$, fluoxetine versus placebo and desvenlafaxine versus placebo. CDRS-R, Children's Depression Rating Scale-Revised; CI, confidence interval; ITT, intent-to-treat; MMRM, mixed-effects model for repeated measures; SE, standard error. 
Table 2. Summary of Secondary Efficacy Outcomes at Week 8

\begin{tabular}{|c|c|c|c|c|c|c|}
\hline$C G I-S$ & & & $\begin{array}{l}\text { ted mean } \\
\text { nge }(S E) \\
\text { baseline }^{\mathrm{a}}\end{array}$ & $\begin{array}{l}\text { Difference in } \\
\text { adjusted means } \\
\text { (placebo-active) }\end{array}$ & $95 \% C I$ & $\mathrm{p}$ \\
\hline Placebo & & & $1(0.12)$ & - & - & - \\
\hline Fluoxetine & & & $8(0.12)$ & 0.18 & -0.11 to 0.46 & 0.224 \\
\hline Desvenlafaxine & & & $0(0.11)$ & -0.01 & -0.29 to 0.27 & 0.944 \\
\hline$C G I-I^{\mathrm{b}}$ & $\mathrm{n}$ & $\begin{array}{c}\text { Very much } \\
\text { improved }(\%)\end{array}$ & $\begin{array}{c}\text { Much } \\
\text { improved (\%) }\end{array}$ & $\begin{array}{c}\text { Minimally } \\
\text { improved (\%) }\end{array}$ & No change (\%) & $\begin{array}{c}\text { CMH test }{ }^{\mathrm{c}} \\
\mathrm{p} \text {-value }\end{array}$ \\
\hline Placebo & 99 & 27.3 & 35.4 & 32.3 & 4.0 & - \\
\hline Fluoxetine & 101 & 30.7 & 47.5 & 16.8 & 4.0 & 0.095 \\
\hline Desvenlafaxine & 99 & 23.2 & 45.5 & 21.2 & 9.1 & 0.852 \\
\hline CGI-I response ${ }^{\mathrm{d}}$ & & & $\%$ & $\begin{array}{c}\text { Adjusted } \\
\text { odds ratio }\end{array}$ & Wald $95 \%$ CI & $\mathrm{p}$ \\
\hline Placebo & & & 62.6 & - & - & - \\
\hline Fluoxetine & & & 78.2 & 0.465 & 0.249 to 0.871 & 0.017 \\
\hline Desvenlafaxine & & & 68.7 & 0.751 & 0.415 to 1.357 & 0.343 \\
\hline
\end{tabular}

${ }^{\mathrm{a}}$ Estimates of adjusted mean, SE, difference in means, $95 \% \mathrm{CI}$, and $p$-values were based on a mixed-effects model for repeated measures for the change from baseline in CGI-S with terms for treatment, week, interaction of treatment and week, age group, country, gender, and baseline CGI-S total score.

${ }^{\mathrm{b}}$ CGI-I scored as 1, very much improved; 2 , much improved; 3 , minimally improved; 4 , no change; 5 , minimally worse; 6 , much worse; 7 , very much worse. No patients scored 5-7 at week 8 .

${ }^{\mathrm{c}} \mathrm{CMH}$ test was controlled for age group and country.

${ }^{\mathrm{d}}$ CGI-I response was defined as CGI-I score of 1 (very much improved) or 2 (much improved).

${ }^{e}$ Estimates of odds ratios, $95 \% \mathrm{CI}$, and $p$-values were based on a logistic regression model of the response rate, with terms for treatment group, age group, and country.

CGI-I, Clinical Global Impressions-Improvement; CGI-S, Clinical Global Impressions-Severity; CI, confidence interval; CMH, Cochran-MantelHaenszel; SE, standard error.

Suicidality. A summary of C-SSRS results for treatmentemergent suicidal ideation or behavior is presented for the safety population in Table 4 (full C-SSRS results are presented in Supplementary Table S3). Treatment-emergent suicidal ideation or suicidal behavior, which included both new-onset and worsening suicidal ideation or behavior, was reported for 29 (8.6\%) of 337 patients who had a C-SSRS assessment at baseline and at 1 or more postbaseline time points. A total of 29/337 (8.6\%) patients had treatment-emergent suicidal ideation, and 1 patient $(0.3 \%)$ also had treatment-emergent suicidal behavior. The suicidal behavior event

Table 3. Number (\%) of Patients Reporting Treatment-Emergent Adverse Events with Incidence $\geq 5 \%$ in Any Group, On-Therapy Period, Safety Population

\begin{tabular}{|c|c|c|c|c|c|c|c|c|c|}
\hline & \multicolumn{3}{|c|}{ Children } & \multicolumn{3}{|c|}{ Adolescents } & \multicolumn{3}{|c|}{ Overall } \\
\hline & $\begin{array}{c}\text { Placebo } \\
(\mathrm{n}=42)\end{array}$ & $\begin{array}{l}\text { Fluoxetine } \\
(\mathrm{n}=45)\end{array}$ & $\begin{array}{l}\text { Desvenlafaxine } \\
\quad(\mathrm{n}=43)\end{array}$ & $\begin{array}{c}\text { Placebo } \\
(\mathrm{n}=70)\end{array}$ & $\begin{array}{l}\text { Fluoxetine } \\
(\mathrm{n}=67)\end{array}$ & $\begin{array}{l}\text { Desvenlafaxine } \\
\quad(\mathrm{n}=72)\end{array}$ & $\begin{array}{l}\text { Placebo } \\
(\mathrm{n}=112)\end{array}$ & $\begin{array}{l}\text { Desvenlafaxine } \\
\quad(\mathrm{n}=112)\end{array}$ & $\begin{array}{l}\text { Desvenlafaxine } \\
\quad(\mathrm{n}=115)\end{array}$ \\
\hline Any TEAE & $27(64.3)$ & $29(64.4)$ & $22(51.2)$ & $52(74.3)$ & 43 (64.2) & $47(65.3)$ & $79(70.5)$ & $72(64.3)$ & $69(60.0)$ \\
\hline Headache & $8(19.0)$ & $3(6.7)$ & 5 (11.6) & 13 (18.6) & $13(19.4)$ & $14(19.4)$ & $21(18.8)$ & $16(14.3)$ & $19(16.5)$ \\
\hline $\begin{array}{l}\text { Abdominal } \\
\text { pain upper }\end{array}$ & $2(4.8)$ & $1(2.2)$ & $6(14.0)$ & $5(7.1)$ & 8 (11.9) & $9(12.5)$ & $7(6.3)$ & $9(8.0)$ & $15(13.0)$ \\
\hline Nausea & $3(7.1)$ & $4(8.9)$ & $2(4.7)$ & $7(10.0)$ & $9(13.4)$ & $6(8.3)$ & $10(8.9)$ & 13 (11.6) & $8(7.0)$ \\
\hline Dizziness & $3(7.1)$ & $1(2.2)$ & $2(4.7)$ & $3(4.3)$ & $2(3.0)$ & $5(6.9)$ & $6(5.4)$ & $3(2.7)$ & $7(6.1)$ \\
\hline Influenza & 0 & $2(4.4)$ & $2(4.7)$ & 0 & 0 & $4(5.6)$ & 0 & $2(1.8)$ & $6(5.2)$ \\
\hline Nasopharyngitis & $4(9.5)$ & $2(4.4)$ & $1(2.3)$ & $4(5.7)$ & $5(7.5)$ & $5(6.9)$ & $8(7.1)$ & $7(6.3)$ & $6(5.2)$ \\
\hline $\begin{array}{l}\text { Upper } \\
\text { respiratory } \\
\text { tract infection }\end{array}$ & $2(4.8)$ & $3(6.7)$ & $1(2.3)$ & $4(5.7)$ & $1(1.5)$ & $5(6.9)$ & $6(5.4)$ & $4(3.6)$ & $6(5.2)$ \\
\hline Vomiting & $1(2.4)$ & $5(11.1)$ & $1(2.3)$ & $3(4.3)$ & $2(3.0)$ & $4(5.6)$ & $4(3.6)$ & $7(6.3)$ & $5(4.3)$ \\
\hline Diarrhea & $1(2.4)$ & 0 & $4(9.3)$ & $2(2.9)$ & $3(4.5)$ & $1(1.4)$ & $3(2.7)$ & $3(2.7)$ & $5(4.3)$ \\
\hline Insomnia & $1(2.4)$ & $1(2.2)$ & 0 & $2(2.9)$ & $4(6.0)$ & $4(5.6)$ & $3(2.7)$ & $5(4.5)$ & $4(3.5)$ \\
\hline Fatigue & $1(2.4)$ & $3(6.7)$ & 0 & $1(1.4)$ & $3(4.5)$ & $2(2.8)$ & $2(1.8)$ & $6(5.4)$ & $2(1.7)$ \\
\hline Pharyngitis & $2(4.8)$ & $3(6.7)$ & $1(2.3)$ & 0 & $1(1.5)$ & $1(1.4)$ & $2(1.8)$ & $4(3.6)$ & $2(1.7)$ \\
\hline Rash & 0 & $3(6.7)$ & 0 & 0 & $1(1.5)$ & $1(1.4)$ & 0 & $4(3.6)$ & $1(0.9)$ \\
\hline Arthralgia & $3(7.1)$ & $1(2.2)$ & $1(2.3)$ & $1(1.4)$ & $2(3.0)$ & 0 & $4(3.6)$ & $3(2.7)$ & $1(0.9)$ \\
\hline Fall & $2(4.8)$ & $4(8.9)$ & 0 & 0 & $1(1.5)$ & 0 & $2(1.8)$ & $5(4.5)$ & 0 \\
\hline Constipation & $3(7.1)$ & 0 & 0 & $1(1.4)$ & 0 & 0 & $4(3.6)$ & 0 & 0 \\
\hline
\end{tabular}

TEAE, treatment-emergent adverse event. 
Table 4. Summary of Treatment-Emergent Suicidal Ideation and Behavior Reported on the Columbia-Suicide Severity Rating Scale at Any Postbaseline Assessment, Safety Population

\begin{tabular}{|c|c|c|c|c|}
\hline & $\begin{array}{l}\text { Placebo } \\
(\mathrm{N}=112)\end{array}$ & $\begin{array}{c}\text { Fluoxetine } \\
(\mathrm{N}=110)\end{array}$ & $\begin{array}{l}\text { Desvenlafaxine } \\
\quad(\mathrm{N}=115)\end{array}$ & $\begin{array}{c}\text { Total } \\
(\mathrm{N}=337)\end{array}$ \\
\hline Treatment-emergent SIB $^{\mathrm{a}}$ & $8 / 112(7.1)$ & $12 / 110(10.9)$ & $9 / 115(7.8)$ & 29/337 (8.6) \\
\hline New-onset SIB ${ }^{\mathrm{b}}$ & $7 / 104(6.7)$ & $10 / 97(10.3)$ & $8 / 102(7.8)$ & $25 / 303(8.3)$ \\
\hline Worsening $\mathrm{SIB}^{\mathrm{c}}$ & $1 / 8(12.5)$ & $2 / 13(15.4)$ & $1 / 13(7.7)$ & $4 / 34(11.8)$ \\
\hline Treatment-emergent SI $^{\mathrm{d}}$ & $8 / 112(7.1)$ & $12 / 110(10.9)$ & $9 / 115(7.8)$ & 29/337 (8.6) \\
\hline New-onset $\mathrm{SI}^{\mathrm{e}}$ & $7 / 104(6.7)$ & $10 / 97(10.3)$ & $8 / 102(7.8)$ & $25 / 303(8.3)$ \\
\hline Wish to be dead & 5 & 4 & 3 & 12 \\
\hline Nonspecific active suicidal thoughts & 1 & 1 & 1 & 3 \\
\hline Active SI with any methods (no plan) without intent to act & 1 & 5 & 4 & 10 \\
\hline Worsening $\mathrm{SI}^{\mathrm{f}}$ & $1 / 8(12.5)$ & $2 / 13(15.4)$ & $1 / 13(7.7)$ & $4 / 34(11.8)$ \\
\hline Shift to nonspecific active suicidal thoughts & 1 & 0 & 0 & 1 \\
\hline Shift to active SI with any methods (no plan) without intent to act & 0 & 1 & 1 & 2 \\
\hline Shift to active SI with specific plan and intent & 0 & 1 & 0 & 1 \\
\hline Treatment-emergent $\mathrm{SB}^{\mathrm{g}}$ & $0 / 112(0.0)$ & $1 / 110(0.9)$ & $0 / 115(0.0)$ & $1 / 337(0.3)$ \\
\hline New-onset $\mathrm{SB}^{\mathrm{h}}$ & $0 / 112(0.0)$ & $1 / 110(0.9)$ & $0 / 115(0.0)$ & $1 / 337(0.3)$ \\
\hline Interrupted attempt & 0 & 1 & 0 & 1 \\
\hline Worsening $\mathrm{SB}^{\mathrm{i}}$ & 0 & 0 & 0 & 0 \\
\hline
\end{tabular}

There was one poststudy suicide attempt reported as a serious adverse event that was not captured on the C-SSRS; C-SSRS was not performed following that event. $N$ represents the number of patients in this analysis, that is, patients who had a baseline and a postbaseline C-SSRS assessment.

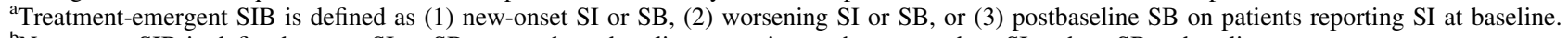

${ }^{\mathrm{b}} \mathrm{New}$-onset SIB is defined as any SI or SB reported postbaseline on patients who reported no SI and no SB at baseline.

${ }^{\mathrm{c}}$ Worsening SIB is defined as (1) shift from SI at baseline to a more severe SI postbaseline, (2) shift from SI at baseline (and no SB at baseline) to any SB postbaseline, or (3) shift from SB at baseline to a more severe SB postbaseline.

${ }^{\mathrm{d}}$ Treatment-emergent SI is defined as new-onset SI or worsening SI.

${ }^{\mathrm{e}} \mathrm{New}$-onset SI is defined as any SI reported postbaseline on patients who reported no SI at baseline.

${ }^{\mathrm{f}}$ Worsening SI is defined as shift to a more severe SI postbaseline on patients reporting SI at baseline.

${ }^{\mathrm{g}}$ Treatment-emergent SB is defined as new-onset SB or worsening SB.

${ }^{\mathrm{h}} \mathrm{New}$-onset SB is defined as any SB reported postbaseline on patients who reported no SB at baseline.

${ }^{\mathrm{i}}$ Worsening SB is defined as shift to a more severe SB postbaseline on patients reporting SB at baseline.

C-SSRS, Columbia-Suicide Severity Rating Scale; SB, suicidal behavior; SI, suicidal ideation; SIB, suicidal ideation or behavior.

was categorized as a preparatory act toward imminent suicidal behavior (preparatory acts or behavior; interrupted attempt) and was reported in a fluoxetine-treated adolescent (Supplementary Table S3). The patient was discontinued due to a serious AE of acute suicidality (described in "Deaths and Serious AEs" section).

New-onset self-injurious behavior without suicidal intent was reported in two (1.8\%) fluoxetine-treated adolescents and one $(0.9 \%)$ desvenlafaxine-treated adolescent.

The suicide attempt reported as a serious AE in the "Deaths and Serious AEs" section was not captured on the C-SSRS because it occurred poststudy.

Other safety measures. The frequencies of patients with ontherapy potentially clinically important (PCI) vital sign values can be found in Supplementary Table S4. Upon review of patient data by the medical monitor, eight patients (all adolescents) were deemed to have clinically important vital sign results: postural hypotension in three desvenlafaxine-treated patients (35-, 35-, and 50-mg/d doses) and in four fluoxetine-treated patients, and increased supine systolic blood pressure in one desvenlafaxine-treated patient $(122-126 \mathrm{~mm}$ $\mathrm{Hg}$ at weeks 3-6; baseline, $117 \mathrm{~mm} \mathrm{Hg}$ ). No patients assigned to placebo had clinically important vital sign findings, and the observed changes from baseline in body mass index (BMI) were negligible. Mean changes from baseline in BMI, blood pressure, and pulse measurements are reported by age group in Supplementary Table S5.

Expected shifts associated with development assessed by Tanner staging were observed during the study. Clinically important ECG findings were reported for one patient assigned to fluoxetine who had Wolff-Parkinson-White syndrome at study entry, with no change over the course of the study.

The frequencies of on-therapy PCI laboratory findings are presented in Supplementary Table S6. Findings in nine patients (four desvenlafaxine, five fluoxetine) were determined to be clinically important: three had elevated triglycerides (two desvenlafaxine, one fluoxetine), one high prolactin (desvenlafaxine), one low hematocrit and low hemoglobin (desvenlafaxine), three positive urine protein (fluoxetine), and one abnormal liver function test (fluoxetine). No clinically important laboratory values were observed in the placebo group. Mean changes from baseline for selected laboratory values are reported by age group and treatment in Supplementary Table S7.

\section{Discussion}

Neither desvenlafaxine nor fluoxetine demonstrated efficacy for treating MDD in children and adolescents in this study. No statistically significant difference between desvenlafaxine and placebo groups, or between fluoxetine and placebo groups, was observed for the primary efficacy endpoint (CDRS-R). At study week 8, patients in desvenlafaxine and placebo treatment groups achieved mean 22.6and 23.1-point improvements from baseline in CDRS-R total score, respectively. However, because fluoxetine (24.8-point improvement) also did not statistically separate from placebo on the primary endpoint, the study meets the Division of Psychiatry Products of the U.S. Food and Drug Administration definition of a failed trial (Food and Drug Administration 2015), and the efficacy results for desvenlafaxine therefore were inconclusive. Desvenlafaxine also failed 
to demonstrate efficacy for treating pediatric MDD in a sister study, which was a negative trial (no active control arm) (Atkinson et al. 2017). Findings for CGI-S (key secondary endpoint) and CGI-I scores in the current study were generally consistent with the primary efficacy results.

The fluoxetine reference arm was included in the current study to provide assay sensitivity because fluoxetine has demonstrated efficacy in children and adolescents with MDD in several placebocontrolled studies (Emslie et al. 1997, 2002). A significant difference between fluoxetine and placebo in improvement on the CDRS-R total score was therefore expected in the current study, although in some recent studies fluoxetine did not demonstrate efficacy in children and adolescents (Atkinson et al. 2014; Emslie et al. 2014). The CGI-I responder rate was the only efficacy measure in this study on which fluoxetine statistically separated from placebo. Because fluoxetine did not separate from placebo on the primary efficacy endpoint, no conclusion can be drawn from the results of this study regarding the efficacy of desvenlafaxine in the treatment of pediatric MDD.

Desvenlafaxine and fluoxetine treatment in this study were associated with mean decreases in CDRS-R scores of 23 and 25 points, respectively, similar to antidepressant response observed in positive pediatric MDD trials (Emslie et al. 1997, 2002, 2009; Wagner et al. 2003, 2004). The placebo response in the current trial ( -23 points), however, was in line with that observed in published negative or inconclusive pediatric depression trials (Emslie et al. 2006, 2007, 2014; Atkinson et al. 2014; DelBello et al. 2014). In a meta-analysis of 12 pediatric antidepressant trials, Bridge et al. (2009) found that placebo response was more predictive of the efficacy outcome of a trial than was study medication response. Factors associated with a larger placebo response in that analysis were greater number of study sites and less severe depression at baseline (Bridge et al. 2009). The number of study sites (37) and the baseline severity of depression (mean CDRS-R total score, 56.5) in the current study were within the ranges reported in positive pediatric antidepressant trials (study sites: 1-53; baseline CDRS-R total scores: 55.1-64.6) (Emslie et al. 1997, 2002, 2009; Wagner et al. 2003, 2004)

Several approaches to minimizing placebo response were used in this study as follows: limited number of treatment arms, rater certification for the CDRS-R, use of the same rater for a given patient whenever possible, and instruction to clinician investigators to refrain from positive emotional response to improvements in symptoms during study assessments or communication of expectancies for improvement. Different approaches to addressing placebo response may vary in their effect on efficacy outcomes, however, and the specific methods used in the current study may not have been sufficient. Study design elements used in positive SSRI/SNRI studies but not in our study included the use of two treatment arms only (study medication and placebo) and 1:1 randomization (Emslie et al. 1997, 2002, 2009; Wagner et al. 2003, 2004), which has been associated with a lower placebo response (Weimer et al. 2015). All but one of those positive studies used a placebo run-in (Emslie et al. 1997, 2002, 2009; Wagner et al. 2003, 2004) and most included multiple diagnostic interviews by independent raters to confirm diagnosis before entry (Emslie et al. 1997, 2002, 2009; Wagner et al. 2004).

Recent thinking about placebo response as ubiquitous in clinical medicine has led to additional recommendations, including systematic measuring and statistical control for nonpharmacological patient factors and therapeutic alliance, separation of recruitment from trial conductance to reduce selection bias by the clinician, and assessment of and controlling for the effectiveness of blinding (Enck et al. 2013; Rief et al. 2016). If implemented, such processes might have reduced the placebo response in this trial or allowed for analyzing its contribution separately from the drug/placebo effects. The increased care and attention from parents and site personnel that is associated with entering a trial and participating in repeated site visits are likely to have contributed to the reduction of symptoms in both groups. Whether this effect is stronger in the placebo than in the active drug group is not known, but recent early results suggest that this may be the case (Zilcha-Mano et al. 2015). Designing future studies to assess and analyze results to determine this difference could help identify pharmacologic effects separate from nonspecific treatment effects (Zilcha-Mano 2016).

The current study provides valuable information regarding the safety and tolerability of desvenlafaxine treatment in a pediatric population. In the previous open-label short-term and extension studies of desvenlafaxine (Findling et al. 2014), desvenlafaxine (children, $10-100 \mathrm{mg} / \mathrm{d}$; adolescents, $25-200 \mathrm{mg} / \mathrm{d}$ ) was generally safe and well tolerated, with AEs similar to those reported in adult studies. Those studies did not include a placebo comparison, however (Findling et al. 2014), limiting conclusions regarding the balance of benefits and risks of desvenlafaxine treatment in pediatric patients (Emslie 2009). In this study, rates of TEAEs were comparable for desvenlafaxine and placebo groups among both children and adolescents, and no statistically significant differences were observed in the incidence of prespecified AEs of clinical interest, including events related to blood pressure or aggressive or suicidal behavior. Although some statistical differences between groups were noted in laboratory evaluations and physical and vital sign measurements, none was considered to be clinically meaningful. The increased number of fluoxetine- versus placebotreated patients with orthostatic hypotension is consistent with previously reported effects (Pacher and Kecskemeti 2004; Jerrell and McIntyre 2009).

New-onset suicidal ideation was reported for 8, 10, and 7 patients in the desvenlafaxine, fluoxetine, and placebo groups, respectively; suicidal behavior was reported for 1 fluoxetine-treated patient who reported suicidal ideation at baseline. No evidence of a signal for increased risk of suicidal thoughts or behaviors has been observed in adults receiving desvenlafaxine treatment for MDD (Tourian et al. 2010). However, for the antidepressant class (SSRIs/ SNRIs), there appears to be a small increased risk of treatmentemergent suicidal ideation/suicide attempt associated with antidepressant treatment of MDD in children and adolescents (1\% risk difference, antidepressant vs. placebo), based on a meta-analysis of 15 trials (Bridge et al. 2007). Bridge et al. (2007) concluded that the overall risk-to-benefit profile for antidepressants in the treatment of pediatric MDD is favorable based on the benefits of treatment; however, the risk/benefit balance may differ across patient populations.

Limitations of the current study should be noted. First, the enrollment criteria selected for a study population that was generally medically healthy, excluding patients at risk for suicide or with co-occurring psychiatric conditions necessitating treatment with medication or other unstable medical conditions, thus limiting generalization of study conclusions to a broader pediatric MDD population. In addition, median CGI-S score at baseline indicated that the majority of patients in the study had moderately severe depression at baseline. Analysis of the effect of baseline severity in adult MDD trials suggests that larger treatment effect size might be observed in patients with more severe baseline depression (Fournier et al. 2010). 


\section{Conclusions}

This phase III, double-blind, placebo-controlled study failed to demonstrate efficacy of desvenlafaxine for treating MDD in children and adolescent patients. No significant difference between placebo- and desvenlafaxine-treated patients was observed for the primary efficacy variable, change from baseline in CDRS-R total scores at week 8. A fluoxetine treatment arm was included in the study because fluoxetine has previously demonstrated efficacy in children and adolescents with MDD. However, because fluoxetine also did not separate from placebo on the primary endpoint in this study, neither medication demonstrated efficacy in the current trial. Therefore, no conclusion can be drawn from these results regarding the efficacy of desvenlafaxine in the treatment of pediatric MDD. Desvenlafaxine was generally safe and well tolerated in children and adolescents in this study. No new safety signals were identified. Safety results were consistent with previous adult and pediatric desvenlafaxine MDD trials.

\section{Clinical Significance}

Untreated depression can affect family interactions, social development and functioning, and school performance in children and adolescents with MDD. However, few antidepressant medications have been approved for this patient population. The efficacy of desvenlafaxine was assessed in a placebo-controlled study that included an active medication (fluoxetine) reference arm. No statistically significant difference was observed between desvenlafaxine and placebo on the primary efficacy endpoint (CDRS-R). Because fluoxetine also did not separate from placebo, no conclusion can be drawn from these results regarding the efficacy of desvenlafaxine in the treatment of pediatric MDD.

\section{Disclosures}

K.L.W.: received compensation from Pfizer Inc for her role as one of the Principal Investigators who conducted this study; received compensation in the past from Pfizer Inc and other pharmaceutical companies for her role as a Principal Investigator in numerous clinical trials; and has been a Pharmacy and Therapeutics consultant for CVS/Caremark. W.M.: received compensation from Pfizer Inc for his role as one of the Principal Investigators who conducted this study; received compensation in the past from Pfizer Inc and other pharmaceutical companies for his role as a Principal Investigator in numerous clinical trials. R.A.: employee of Pfizer Inc. D.C.: employee of Pfizer Inc and has Pfizer Inc stock options. R.D.E.: employee of Pfizer Inc, owns Pfizer Inc stock, and has Pfizer Inc stock options. S.R.: employee of Pfizer Inc, owns Pfizer Inc stock, and has Pfizer Inc stock options. D.B.W.: employee of Pfizer Inc, owns Pfizer Inc stock, and has Pfizer Inc stock options.

\section{Acknowledgments}

Medical writing support was provided by Kathleen M. Dorries, $\mathrm{PhD}$, of Peloton Advantage and funded by Pfizer Inc. The authors thank the patients and family members who contributed their time to this study. Site investigators, coordinators, as well as study monitors, data managers, and data analysts were essential to the accomplishment of this trial and are appreciated by the authors. Dalia B. Wajsbrot (Pfizer Inc) served as the statistical expert for this study. Investigators who enrolled five or more patients included: Gustavo Alva, Valerie Kaplan Arnold, Ashraf Mohamed Attalla, Louise Marion Beckett-Thurman, Jesse Mack Carr, Ann Catherine Childress, Daniel F. Chueh, Ariel De Llanos, Douglas Robert
Dolnak, Andro Giorgadze, Nelson Manuel Handal-Thome, Joseph Allen Kwentus, Gregory W. Mattingly, Paul Richard Miller, Marino Molina Jr., Robert Bond Molpus, Eliot Moon, William Rory Murphy, Kambiz Pahlavan, William Jeffrey Palmer, Randall Kenneth Ricardi, Robert Alan Riesenberg, Erasmo Saucedo-Uribe, Charmaine Irene Semeniuk, Jerry Carl Steiert, Ekaterina Vassilenko, Juan Luis Vazquez-Hernandez, Marianne Zdeblick Wamboldt, Karen Louise Weihs, Kashinath Gangadhara Yadalam, and Alan Soon Yeo. The sponsor was involved in the design of the study and the collection (through contracted study site investigators), analysis, and interpretation of study data. Pfizer Inc authors contributed to the writing of the report and the decision to submit the article for publication.

\section{References}

American Psychiatric Association: Diagnostic and Statistical Manual of Mental Disorders, 4th ed., Text Revision. Washington, DC: American Psychiatric Association; 2000.

Atkinson S, Lubaczewski S, Ramaker S, England RD, Wajsbrot DB, Abbas R, Findling RL: Desvenlafaxine versus placebo in the treatment of children and adolescents with major depressive disorder. J Child Adolesc Psychopharmacol 2017;28:55-65.

Atkinson SD, Prakash A, Zhang Q, Pangallo BA, Bangs ME, Emslie GJ, March JS: A double-blind efficacy and safety study of duloxetine flexible dosing in children and adolescents with major depressive disorder. J Child Adolesc Psychopharmacol 24:180-189, 2014.

Birmaher B, Brent D, Bernet W, Bukstein O, Walter H, Benson RS, Chrisman A, Farchione T, Greenhill L, Hamilton J, Keable H, Kinlan J, Schoettle U, Stock S, Ptakowski KK, Medicus J: Practice parameter for the assessment and treatment of children and adolescents with depressive disorders. J Am Acad Child Adolesc Psychiatry 46:1503-1526, 2007.

Birmaher B, Ryan ND, Williamson DE, Brent DA, Kaufman J, Dahl RE, Perel J, Nelson B: Childhood and adolescent depression: A review of the past 10 years. Part I. J Am Acad Child Adolesc Psychiatry 35:1427-1439, 1996.

Boyer P, Montgomery S, Lepola U, Germain JM, Brisard C, Ganguly R, Padmanabhan SK, Tourian KA: Efficacy, safety, and tolerability of fixed-dose desvenlafaxine 50 and $100 \mathrm{mg} /$ day for major depressive disorder in a placebo-controlled trial. Int Clin Psychopharmacol 23:243-253, 2008.

Bridge JA, Birmaher B, Iyengar S, Barbe RP, Brent DA: Placebo response in randomized controlled trials of antidepressants for pediatric major depressive disorder. Am J Psychiatry 166:42-49, 2009.

Bridge JA, Iyengar S, Salary CB, Barbe RP, Birmaher B, Pincus HA, Ren L, Brent DA: Clinical response and risk for reported suicidal ideation and suicide attempts in pediatric antidepressant treatment: A meta-analysis of randomized controlled trials. JAMA 297:16831696, 2007.

Chan IS, Zhang Z: Test-based exact confidence intervals for the difference of two binomial proportions. Biometrics 55:12021209, 1999.

Cheung AH, Zuckerbrot RA, Jensen PS, Ghalib K, Laraque D, Stein RE: Guidelines for Adolescent Depression in Primary Care (GLAD-PC): II. Treatment and ongoing management. Pediatrics 120: e1313-e1326, 2007.

Clayton A, Tourian KA, Focht K, Hwang E, Cheng R-FJ, Thase ME: Desvenlafaxine 50- and $100-\mathrm{mg} / \mathrm{d}$ vs placebo for the treatment of major depressive disorder: A phase 4, randomized controlled trial. J Clin Psychiatry 76:562-569, 2015.

Clayton AH, Kornstein SG, Dunlop BW, Focht K, Musgnung J, Ramey T, Bao W, Ninan PT: Efficacy and safety of desvenlafaxine 
$50 \mathrm{mg} / \mathrm{d}$ in a randomized, placebo-controlled study of perimenopausal and postmenopausal women with major depressive disorder. J Clin Psychiatry 74:1010-1017, 2013.

Clayton AH, Kornstein SG, Rosas G, Guico-Pabia C, Tourian KA: An integrated analysis of the safety and tolerability of desvenlafaxine compared with placebo in the treatment of major depressive disorder. CNS Spectr 14:183-195, 2009.

DelBello MP, Hochadel TJ, Portland KB, Azzaro AJ, Katic A, Khan A, Emslie G: A double-blind, placebo-controlled study of selegiline transdermal system in depressed adolescents. J Child Adolesc Psychopharmacol 24:311-317, 2014.

Demartinis NA, Yeung PP, Entsuah R, Manley AL: A double-blind, placebo-controlled study of the efficacy and safety of desvenlafaxine succinate in the treatment of major depressive disorder. J Clin Psychiatry 68:677-688, 2007.

Dunlop BW, Reddy S, Yang L, Lubaczewski S, Focht K, Guico-Pabia $\mathrm{CJ}$ : Symptomatic and functional improvement in employed depressed patients: A double-blind clinical trial of desvenlafaxine versus placebo. J Clin Psychopharmacol 31:569-576, 2011.

Emslie GJ: Understanding placebo response in pediatric depression trials. Am J Psychiatry 166:1-3, 2009.

Emslie GJ, Findling RL, Yeung PP, Kunz NR, Li Y: Venlafaxine ER for the treatment of pediatric subjects with depression: Results of two placebo-controlled trials. J Am Acad Child Adolesc Psychiatry 46:479-488, 2007.

Emslie GJ, Heiligenstein JH, Wagner KD, Hoog SL, Ernest DE, Brown E, Nilsson M, Jacobson JG: Fluoxetine for acute treatment of depression in children and adolescents: A placebo-controlled, randomized clinical trial. J Am Acad Child Adolesc Psychiatry 41: 1205-1215, 2002.

Emslie GJ, Prakash A, Zhang Q, Pangallo BA, Bangs ME, March JS: A double-blind efficacy and safety study of duloxetine fixed doses in children and adolescents with major depressive disorder. J Child Adolesc Psychopharmacol 24:170-179, 2014.

Emslie GJ, Rush AJ, Weinberg WA, Kowatch RA, Hughes CW, Carmody T, Rintelmann J: A double-blind, randomized, placebocontrolled trial of fluoxetine in children and adolescents with depression. Arch Gen Psychiatry 54:1031-1037, 1997.

Emslie GJ, Ventura D, Korotzer A, Tourkodimitris S: Escitalopram in the treatment of adolescent depression: A randomized placebocontrolled multisite trial. J Am Acad Child Adolesc Psychiatry 48: 721-729, 2009.

Emslie GJ, Wagner KD, Kutcher S, Krulewicz S, Fong R, Carpenter DJ, Lipschitz A, Machin A, Wilkinson C: Paroxetine treatment in children and adolescents with major depressive disorder: A randomized, multicenter, double-blind, placebo-controlled trial. J Am Acad Child Adolesc Psychiatry 45:709-719, 2006.

Enck P, Bingel U, Schedlowski M, Rief W: The placebo response in medicine: Minimize, maximize or personalize? Nat Rev Drug Discov 12:191-204, 2013.

Findling RL, Groark J, Chiles D, Ramaker S, Yang L, Tourian KA: Safety and tolerability of desvenlafaxine in children and adolescents with major depressive disorder. J Child Adolesc Psychopharmacol 24:201-209, 2014.

Findling RL, Groark J, Tourian KA, Ramaker SA, Chiles D, Yang L, Nichols AI: Pharmacokinetics and tolerability of single-ascending doses of desvenlafaxine administered to children and adolescents with major depressive disorder. J Child Adolesc Psychopharmacol 26:909-921, 2016.

Food and Drug Administration: Briefing document for the December 1, 2015 Meeting of the Psychopharmacologic Drugs Advisory Committee (PDAC). 2015. www.fda.gov/downloads/AdvisoryCommittees/ CommitteesMeetingMaterials/Drugs/PsychopharmacologicDrugs AdvisoryCommittee/UCM474540.pdf (accessed August 18, 2017).
Fournier JC, Derubeis RJ, Hollon SD, Dimidjian S, Amsterdam JD, Shelton RC, Fawcett J: Antidepressant drug effects and depression severity: A patient-level meta-analysis. JAMA 303:4753, 2010.

Guy W: Clinical Global Impressions. ECDEU Assessment Manual for Psychopharmacology. Rockville, MD, US Department of Health, Education, and Welfare, 1976, pp. 217-222.

Hedeker D, Gibbons RD: Missing Data in Longitudinal Studies. Longitudinal Data Analysis. Hoboken, NJ, John Wiley \& Sons, Inc., 2006, pp. 279-312.

International Council for Harmonisation: ICH harmonised tripartite guideline: Statistical principles for clinical trials E9. 1998. www.ich .org/products/guidelines/efficacy/efficacy-single/article/statisticalprinciples-for-clinical-trials.html (accessed May 15, 2017).

Iwata N, Tourian KA, Hwang E, Mele L, Vialet C, For the Study 3359 Investigators: Efficacy and safety of desvenlafaxine 25 and $50 \mathrm{mg} /$ day in a randomized, placebo-controlled study of depressed outpatients. J Psychiatr Pract 19:5-14, 2013.

Jerrell JM, McIntyre RS: Cardiovascular and neurological adverse events associated with antidepressant treatment in children and adolescents. J Child Neurol 24:297-304, 2009.

Kobak KA, Lipsitz JD, Williams JB, Engelhardt N, Bellew KM: A new approach to rater training and certification in a multicenter clinical trial. J Clin Psychopharmacol 25:407-412, 2005.

Lexapro [package insert]: St. Louis, MO, Forest Pharmaceuticals, Inc., 2014.

Liebowitz MR, Manley AL, Padmanabhan SK, Ganguly R, Tummala $R$, Tourian KA: Efficacy, safety, and tolerability of desvenlafaxine $50 \mathrm{mg} /$ day and $100 \mathrm{mg} /$ day in outpatients with major depressive disorder. Curr Med Res Opin 24:1877-1890, 2008.

Liebowitz MR, Tourian KA, Hwang E, Mele L, For the Study 3362 Investigators: A double-blind, randomized, placebo-controlled study assessing the efficacy and tolerability of desvenlafaxine 10 and $50 \mathrm{mg} / \mathrm{d}$ in adult outpatients with major depressive disorder. BMC Psychiatry 13:94, 2013.

Pacher P, Kecskemeti V: Cardiovascular side effects of new antidepressants and antipsychotics: New drugs, old concerns? Curr Pharm Des 10:2463-2475, 2004.

Posner K, Brown GK, Stanley B, Brent DA, Yershova KV, Oquendo MA, Currier GW, Melvin GA, Greenhill L, Shen S, Mann JJ: The Columbia-Suicide Severity Rating Scale: Initial validity and internal consistency findings from three multisite studies with adolescents and adults. Am J Psychiatry 168:1266-1277, 2011.

Poznanski EO, Cook SC, Carroll BJ: A depression rating scale for children. Pediatrics 64:442-450, 1979.

Pristiq [package insert]: Philadelphia, PA, Wyeth Pharmaceuticals, Inc, a subsidiary of Pfizer Inc, 2016.

Prozac [package insert]. Indianapolis, IN, Eli Lilly and Company, 2014.

Rief W, Barsky AJ, Bingel U, Doering BK, Schwarting R, Wohr M, Schweiger U: Rethinking psychopharmacotherapy: The role of treatment context and brain plasticity in antidepressant and antipsychotic interventions. Neurosci Biobehav Rev 60:51-64, 2016.

Septien-Velez L, Pitrosky B, Padmanabhan SK, Germain JM, Tourian KA: A randomized, double-blind, placebo-controlled trial of desvenlafaxine succinate in the treatment of major depressive disorder. Int Clin Psychopharmacol 22:338-347, 2007.

Siu AL: Screening for depression in children and adolescents: U.S. Preventive Services Task Force recommendation statement. Ann Intern Med 164:360-366, 2016.

Thase ME, Kornstein SG, Germain JM, Jiang Q, Guico-Pabia C, Ninan PT: An integrated analysis of the efficacy of desvenlafaxine compared with placebo in patients with major depressive disorder. CNS Spectr 14:144-154, 2009. 
Tourian KA, Padmanabhan SK, Groark J, Brisard C, Farrington D: Desvenlafaxine 50 and $100 \mathrm{mg} / \mathrm{d}$ in the treatment of major depressive disorder: An 8-week, phase III, multicenter, randomized, double-blind, placebo-controlled, parallel-group trial and a post hoc pooled analysis of three studies. Clin Ther 31 Pt 1:1405-1423, 2009.

Tourian KA, Padmanabhan K, Groark J, Ninan PT: Retrospective analysis of suicidality in patients treated with the antidepressant desvenlafaxine. J Clin Psychopharmacol 30:411-416, 2010.

Wagner KD, Ambrosini P, Rynn M, Wohlberg C, Yang R, Greenbaum MS, Childress A, Donnelly C, Deas D: Efficacy of sertraline in the treatment of children and adolescents with major depressive disorder: Two randomized controlled trials. JAMA 290:1033-1041, 2003.

Wagner KD, Robb AS, Findling RL, Jin J, Gutierrez MM, Heydorn WE: A randomized, placebo-controlled trial of citalopram for the treatment of major depression in children and adolescents. Am J Psychiatry 161:1079-1083, 2004.
Weimer K, Colloca L, Enck P: Placebo effects in psychiatry: Mediators and moderators. Lancet Psychiatry 2:246-257, 2015.

Zilcha-Mano S: New analytic strategies help answer the controversial question of whether alliance is therapeutic in itself. World Psychiatry 15:84-85, 2016.

Zilcha-Mano S, Roose SP, Barber JP, Rutherford BR: Therapeutic alliance in antidepressant treatment: Cause or effect of symptomatic levels? Psychother Psychosom 84:177-182, 2015.

Address correspondence to: Karen L. Weihs, MD Department of Psychiatry University of Arizona 1501 N. Campbell Avenue No. 7306D

Tucson, AZ 85724-5002

E-mail: weihs@psychiatry.arizona.edu 